\title{
Jan Foitzik
}

\section{Sowjetische Hegemonie und Ostintegration der DDR}

Der „Ostblock“ gehört zu den vernachlässigten Bereichen der Historiographie. Eine Vorstellung über die Tragfähigkeit des gegenwärtigen Wissensstandes sollen einige Zitate aus der Tagespresse illustrieren. Darin hieß es ursprünglich, daß die DDR ihre staatliche Existenz ausweislich ihrer Außenhandelsbilanz mit der UdSSR mit einem Defizit abgeschlossen habe und erst bei der Vorbereitung der deutschen Währungsunion herausgekommen sei, daß die DDR keine Schulden gegenüber der UdSSR hatte. Im Frühjahr 2001 - nach zehn Jahren also - lauteten die Schlagzeilen, daß Rußland aufgrund alter DDR-Außenhandelsbilanzen der Bundesrepublik etwa zwei Milliarden DM schulde. Diese Summe war in der russischen Schuldenbilanz nicht enthalten, letztlich akzeptierte sie aber die russische Regierung. Die Fachwissenschaft schlägt einen autonomen Weg ein und geht von einem Exportüberschuß der DDR von ca. zwei Milliarden Transferrubel Mitte 1990 aus, die mit etwa fünf Milliarden DM zu Buche schlagen. ${ }^{1}$

Außerhalb der Ebene der deutsch-russischen Beziehungen ist die Situation noch unübersichtlicher. So galten noch 1989/90 Polen und Ungarn als Nutznießer der ökonomischen Kooperation mit der UdSSR, während in tschechischen Medien die gesamten Kooperationslasten neuerdings in US-Dollar mit einer dreistelligen Milliardensumme beziffert werden. Politisch wären solche Summen auch dann relevant, wenn sich die nicht benannte Bewertungsmethode als falsch herausstellen sollte.

Erhebliche Einordnungsprobleme bereiten aber auch die wenigen bisher bekannt gewordenen Details: So weisen etwa die Angaben der veröffentlichten DDR-Außenhandelsstatistik über den Handel mit der UdSSR im Jahr 1950 gegenüber internen sowjetischen Papieren einen Mehrwert von etwa 300 Millionen Mark aus, was einem Viertel des Umsatzes entsprach. Sowjetische Papiere wiederum bilanzieren den SBZ-Handel mit der UdSSR für 1949 mit einem Defizit von einer halben Milliarde Mark.

Angesichts der Größe solcher „weißen Flecken" beschränke ich mich im kleinen Rahmen der gestellten Aufgabe darauf, unter politikgeschichtlicher Fragestellung einige thematisch wichtige Punkte zu markieren. Im Zentrum stehen zwei Strukturmomente. Zunächst soll die politisch-kulturelle Dimension der Ostintegration der DDR gestreift werden, die von ihrer Gesamtwirkung her sicherlich einen nationalgeschichtlichen Stellenwert hatte; danach wird kursorisch der Prozeß der politischen und ökonomischen Integration der DDR in das sowjetische Hegemonialsystem thematisiert. Weitgehend beiseite gelassen wird die Deutschlanddiplomatie und kurzerhand hypothetisch davon ausgegangen, daß die Erfahrungen, die vielfältigen und zunehmend perfektionierten Kontroll- und Einflußmöglichkeiten der

1 Vgl. Schwarzer, Sozialistische Zentralplanwirtschaft, S. 220. 
beiden Supermächte sowie das zwischen ihnen durch wechselseitige Kontrolle wachsende Vertrauen ausreichten, um sich in Europa den Besitzstand wechselseitig zu garantieren und Deutschland als ein lokales Problem zu isolieren, das durch den Doppelgriff der NATO und des Warschauer Paktes hinreichend "gedeckelt“ wurde. Außenminister Dulles formulierte die essentials dieser Stabilitätspolitik gegenüber dem sowjetischen Außenminister Gromyko im Oktober 1957 unter vier Augen mit den Worten, daß die USA die mit der deutschen Frage verbundenen Sicherheitsinteressen der UdSSR respektierten und bemüht sein würden, keine Lösung der deutschen Frage anzustreben, die die Sicherheit der UdSSR gefährden oder die NATO stärken könnte ${ }^{2}$. In einer Zusammenfassung werden schließlich in systematisierender Absicht einige Grundmomente des Integrationsprozesses skizziert, von denen zumindest einige weitere Beachtung verdienen.

Auf eine Tiefendurchdringung muß aber nicht nur wegen der skizzierten datentechnischen Einbruchgefahr verzichtet werden, sondern auch mit Blick auf den vorgegebenen zeitlichen Rahmen. Er enthält zwar die Zäsur von 1955/56, als die völkerrechtliche Festschreibung der europäischen Teilung durch Beitritt der beiden deutschen Staaten zur NATO bzw. zum Warschauer Pakt und die Bilder von der sowjetischen Intervention in Budapest am Jahresende 1956 in der öffentlichen Meinung Westdeutschlands Hoffnungen auf eine „österreichische Lösung" für Deutschland zunichte machten ${ }^{3}$ und für Osteuropa die endgültige Bestätigung dafür brachten, daß Moskau und Washington mit „Entstalinisierung“ bzw. „Befreiung vom Kommunismus" nur eine Politik der leeren Worte betrieben. Spätestens nach der Intervention in der ČSSR 1968 führte dies zu der Erkenntnis, daß eine Schwächung der hegemonialen Kontrolle der UdSSR und eine Änderung der bestehenden politischen Verhältnisse nur schrittweise durch eigene Anstrengungen erreicht werden könne. Die Schwerpunktsetzung auf die fünfziger Jahre berücksichtigt aber nicht, daß die völkerrechtliche und ideologische Integration der DDR in den „Ostblock“ erst in der ersten Hälfte der sechziger Jahre vollzogen werden konnte und diese Verzögerung ursächlich mit Schwierigkeiten dieser Integration zusammenhing.

\section{Der politisch-kulturelle Aspekt}

Am 11. Mai 1950 gratulierte das ZK der KPdSU(B) dem ZK der SED aus Anlaß des Jahrestages der „Befreiung des deutschen Volkes vom Hitler-Faschismus“4. Einen inhaltsgleichen Glückwunsch schickte der Vorsitzende der sowjetischen Regierung

2 1957, [rijen], Moskwa. - Informace UV KSSS o rozhovoru ministra zahranici SSSR A. A. Gromyka se statnim tajemnikem USA J. F. Dullesem, in: Reiman/Lunak, Studena valka, S. 55-70, hier S. 65.

3 Noch Anfang der fünfziger Jahre bekannten sich in Westdeutschland bis zu 50\% der Befragten zur außenpolitischen Neutralität, etwas niedriger wurde im Durchschnitt die transatlantische Option präferiert, die im Juni 1953 auf die Zustimmung von 50\% der Befragten hochschnellte.

4 Vgl. Telegramma Zentralnowo Komiteta Kommunistitscheskoi partii Sowetskowo Sojusa Zentralnomu Prawleniju Sozialistitscheskoi jedinoi partii Germanii po slutschaju godowschtiny oswoboschdenija nemezkowo naroda ot gitlerowskogo faschisma vom 11.5. 1950, in: Ministerstwo inostrannych del SSSR/Ministerstwo inostrannych del GDR: Otnoschenija SSSR s GDR, S. 80. 
auch Ministerpräsident Grotewohl ${ }^{5}$. Die von der SED-Partei- und DDR-Staatsführung in ihren Telegrammen an Stalin vom 7. Mai 1950 dem „Vater der Völker" insinuierte $^{6}$ und nach Stalins Salvierung amtlich festgeschriebene Floskel vom HitlerFaschismus $^{7}$ war in zweifacher Hinsicht neu, denn „Befreiung“ war nach Kriegsende in der SBZ/DDR nur als deutsche Ambition artikuliert und von der sowjetischen Zensur strikt unterbunden worden; außerdem war nun das deutsche Volk vom unspezifischen Hitler-Faschismus befreit worden, dessen sozialer Träger zwar parteiabstrakt feststand, dessen nationaler Träger aber im Dunkeln gehalten wurde. Noch ein Jahr zuvor war der 9. Mai in der SBZ als offizieller Gedenktag an den „Zusammenbruch des volksfeindlichen Regimes des aggressiven Hitler-Deutschland" begangen worden, wie die Schlagzeilen der Tagespresse belegen. Vergleicht man beide Phrasen, so ergeben sich insgesamt mehrere Akzentverschiebungen.

Mit diesem voluntaristischen Exkulpationsakt tabuisierte Stalin 1950 die Kollektivschuld-These, auf der die alliierte Nachkriegspolitik gegenüber Deutschland bis zur Verabschiedung des Grundgesetzes im wesentlichen aufbaute. Bei der Differenzierung zwischen der DDR und Deutschland handelte es sich um einen semantischen Kraftakt. In der Sowjetunion selbst blieb das Theorem von der historisch-genetischen Aggressivität der Deutschen, das man 1948 aus dem nationalrussischen Volksschatz hervorgeholt hatte, weiter in Kraft. Um eine deklaratorische Vorleistung handelte es sich auch deshalb, weil Stalins Erklärung von 1950 erst nach Beendigung des Kriegszustandes zwischen der UdSSR und Deutschland im Jahr 1955 erste tatsächliche Lockerungen nach sich zog, als die letzten deutschen Kriegsgefangenen und zivilen $Z$ wangsverschleppten aus sowjetischem Gewahrsam entlassen wurden. Erst 1964 wurden dann bekanntlich die 1941 deportierten Wolga-Deutschen vom Vorwurf der "Kollektivschuld“ moralisch freigesprochen, ohne später je völlig rehabilitiert zu werden. Von historischem Ballast ist das deutsch-russische Verhältnis noch heute nicht frei. Noch 1999 bemühte ein russischer Historiker formalrechtliche Argumente, als er im Zusammenhang mit der Beendigung des Kriegszustandes 1955 Chruschtschow politischen "Subjektivismus" vorwarf und diesen Akt des einseitigen Rechtsverzichts gegenüber der DDR mit dem Moskauer Vertrag von 1970 gleichsetzte, in dem diese Preisgabe von Rechtspositionen ebenso "ohne Not“ auch gegenüber der Bundesrepublik ausgesprochen worden sei ${ }^{8}$.

Über das kulturelle Verhältnis der ostmitteleuropäischen Völker zu Deutschland liegen aus der Zeit nach dem Zweiten Weltkrieg bisher vorwiegend publizistische Äußerungen vor'. Die Ausnahme bildet eine 1987 erschienene polnische Untersuchung über das Deutschenbild in der Nachkriegszeit ${ }^{10}$. Reaktiviert wurde nach dem Krieg in Polen die aus dem 19. Jahrhundert stammende säkularisierte Vorstellung von der eigenen Nationalgeschichte als tausend Jahre politischer und kultureller Auseinandersetzung mit der "deutschen Expansion“. Neu kam hinzu, daß die ur-

5 Ebenda, S. 81.

6 Vgl. ebenda, sowie S. 76 und S. 77.

7 Vgl. Telegramm der DDR-Regierung an Stalin vom 8. 5. 1951, in: Dokumente zur Außenpolitik der DDR, Bd. I, S. $249 \mathrm{f}$. Im Antworttelegramm Stalins war sogar von einer "Befreiung Deutschlands vom faschistischen Joch“ die Rede, ebenda, S. 250.

8 Pochlebkin, Welikaja wojna, S. $361 \mathrm{f}$.

9 Dabei wird von dem Genre der offiziösen Propaganda-Literatur abgesehen.

10 Dmitrow, Niemcy i okupacja Hitlerowska. 
sprünglich als kulturelle Differenz interpretierte Distanz nach dem Krieg auf anthropologische Determinanten zurückgeführt und ein „absoluter Gegensatz zwischen dem Eigenen und dem Fremden " konstruiert wurde, wie dies in jüngster Zeit eine russische Autorin in modischem Klischee ausdrückte ${ }^{11}$. Diese Geschichtsphilosophie war nicht spezifisch polnisch, und ihre Wirkung blieb nicht auf die ersten Nachkriegsjahre beschränkt. Weitgehend verallgemeinerungsfähig war auch die Feststellung, wonach „der Antigermanismus in den ersten Nachkriegsjahren eine wichtige integrative Rolle erfüllte" und in der tief gespaltenen Gesellschaft als Hilfsbrücke fungierte, oder das aufgestellte Junktim zwischen „Hitlerismus“ und Deutschtum. Der polnische Autor fokussiert die Aufmerksamkeit zuletzt auf die Zensur, die nach einer kurzen Phase der freien und durchaus differenzierenden Meinungsäußerung in Polen ein offizielles Deutschen- und Deutschlandbild verbindlich vorschrieb und aufrechterhielt. Im Zentrum dieses neu installierten Stereotyps stand das nationalsozialistische Vernichtungslager als sinnbildlicher Ausdruck der deutschen Geschichte und als "Miniatur der kapitalistischen Gesellschaft“. Das Deutschenbild diente mit anderen Worten ausschließlich als Negativfolie des eigenen kulturellen und politischen Selbstverständnisses.

Im politischen Kontext müssen solche Stereotypen schon deshalb unbedingt als Determinanten beachtet werden, weil entsprechende suggestive Detailaufnahmen über Zensur, Literatur und Geschichtsschreibung zentral verwaltet wurden; außerdem erfüllten sie vielfältige innenpolitische und identitätsstiftende Funktionen. In der sowjetischen Wahrnehmung ist der herausragende Einfluß der kulturellen Komponente auf die eigene Deutschlandpolitik bereits 1945 registriert worden. Das Dilemma zwischen einem historisch schwer belasteten Deutschenbild und einer durch ideologische bzw. pragmatische Überlegungen geleiteten Deutschlandpolitik war jedoch kurzfristig nicht auflösbar, und Moskau blieb ihm weitgehend hilflos ausgeliefert. Auch das 1950 verkündete neue Stereotyp des nationalabstrakten „Faschisten" trug politisch noch lange nicht. Nicht nur in der informellen Wahrnehmung, sondern auch in der Tagespublizistik, in der Literatur und in Schulbüchern handelte es sich in den fünfziger Jahren in Osteuropa nur um ein Synonym, das gleichberechtigt mit deutsch, Deutscher, Deutschland benutzt wurde. Auch in der amtlichen Erklärung über die Beendigung des Kriegszustandes wurde 1955 wieder die Formel „Hitler-Deutschland" benutzt, die etymologisch aus der Zeit vor 1950 stammt. Erst in den sechziger Jahren lösten sich die öffentlichen Bilder über Deutsche langsam von den historischen Belastungen und den politisch-ideologischen Schablonen. Das Schlagwort vom „Faschisten“ konnte nun als leere Phrase seine integrative Wirksamkeit entfalten und dazu beitragen, gegenüber der DDR als einem deutschen Staat immer wieder auftretende Aversionen, Animositäten und Irritationen unter den Teppich zu kehren.

Welche Rolle bei der Überwindung der alten Stereotype das zweite Tabu spielte, das zum ersten Mal im Beschluß des ZK der SED zum Slansky-Prozeß in der Öffentlichkeit auftauchte, sei dahingestellt. Wörtlich hieß es darin: „Für die DDR [...] ist die Vernichtung der Slansky-Banditen [...] von außerordentlicher Bedeutung. Ein Gelingen dieser Pläne hätte die DDR in eine sehr gefährliche Lage gebracht. " ${ }^{12}$

11 Senjawskaja, Obras Germanii i nemzew, S. 11-48.

12 Vgl. Lehren aus dem Prozeß gegen das Verschwörerzentrum Slansky, in: ND vom 4. 2. 1953. 
Diese Erklärung wurde am 19. Dezember 1952 fertiggestellt, also zwei Wochen nach der Massenhinrichtung in Prag, und am 4. Februar 1953 im „Neuen Deutschland“ veröffentlicht ${ }^{13}$, wenige Wochen vor Stalins Tod also. Nur ein halbes Jahr später wurde unter der gleichen Anschuldigung das für allmächtig gehaltene Mitglied der damaligen sowjetischen "Führungstroika“ Berija festgenommen und im Dezember 1953 ebenfalls hingerichtet.

Mit der Propagierung des Vorwurfs der angeblichen Preisgabe der DDR durch Berija und seine Helfershelfer entstand ein selbst die engste politische Führung unmittelbar bindendes Junktim, das in Verbindung mit der nach dem 17. Juni 1953 propagandistisch geschürten Furcht vor einem in Deutschland aktiven nationalsozialistischen Untergrund als Eckpunkt der in Osteuropa amtlich veröffentlichten Meinung diente. Entsprechend den Vorgaben der Hegemonialmacht setzte von nun an die eigene Besitzstandswahrung im Hinblick auf die „Ergebnisse des zweiten Weltkriegs" die Existenz der DDR voraus.

Das konfliktträchtige Dilemma zwischen einem aus der Konstruktion der eigenen historischen Identität abgeleiteten bzw. politisch-propagandistisch erzeugten Mißtrauen gegenüber Deutschen auf der einen und der Pflicht zum Schutz der DDR auf der anderen Seite legte sich erst, nachdem die DDR zu existieren aufgehört hatte. Kulturelle Kontakte mit osteuropäischen Ländern vereinbarte Ost-Berlin schon 1950. Der Kulturaustausch wurde zwar aus politischen Motiven stark forciert, doch zunächst nur halbherzig realisiert. In seinem Zentrum standen zunächst die alljährlich zelebrierten Monate der deutsch-sowjetischen Freundschaft; 1949 wurde der erstmals stattfindende Freundschaftsmonat sogar auf fast zwei Kalendermonate ausgedehnt ${ }^{14}$. Aus dem 1950 in der DDR begangenen „Tag der deutsch-polnischen Freundschaft" sind zwar 1951 und 1952 ebenfalls Monate geworden, doch offenbar stieß die deutsch-polnische Freundschaft in der DDR auf Desinteresse: Im April 1955 beschränkte man sich beispielsweise auf eine Woche. In Polen fand im November 1955 eine „Woche der Freundschaft mit dem demokratischen Deutschland" statt, erst für 1958 ist eine deutsch-tschechoslowakische Freundschaftswoche belegt. In Prag und Berlin entstanden 1955/1956 erste Kulturzentren.

\section{Politische Integration unter dem Vorzeichen der sowjetischen Deutschlandpolitik}

Die erste Phase der Integration der DDR in den „Ostblock“ stand im Zeichen der sowjetischen Deutschlandpolitik. Im Görlitzer Abkommen vom 6. Juli 1950 erkannte die DDR die bestehenden Grenzen, einschließlich der Oder-Neiße-Linie, und in einer gemeinsamen Regierungsdeklaration mit der ČSR die Vertreibung der Deutschen aus der ČSR vorbehaltlos an. Damit war die Oder-Neiße-Grenze zum ersten $\mathrm{Mal}$ in völkerrechtlicher Form fixiert worden. Nachdem die DDR im September 1950 in den seit anderthalb Jahren bestehenden Rat für gegenseitige Wirt-

${ }^{13}$ Sogar in internen tschechoslowakischen und russischen Quellen fehlt jeder Hinweis in dieser Richtung. Unter den Hingerichteten befanden sich auch der erste diplomatische Vertreter der ČSR in der DDR und mehrere Mitarbeiter des Prager Außenministeriums.

14 Vom 30. 10. bis 21. 12. 1949. 
schaftshilfe aufgenommen worden war, traten im Oktober 1950 in Prag die Außenminister Albaniens, Bulgariens, der ČSR, Polens, Rumäniens, Ungarns, der UdSSR und der DDR zusammen, um unter Berufung auf das Potsdamer Protokoll von 1945 gegen die im Zeichen des Koreakrieges beginnende Westintegration Westdeutschlands zu protestieren. Das Außenministertreffen stand in der Tradition der im Februar 1948 auf polnische Initiative in Prag stattfindenden Konferenz der Außenminister Polens, Jugoslawiens und der ČSR sowie des von der sowjetischen Diplomatie initiierten Warschauer Außenministertreffens vom Juni 1948, an dem noch alle acht ${ }$ volksdemokratischen" Staaten (einschließlich Jugoslawiens also) teilgenommen hatten. Die DDR sprang 1950 in die Lücke, die Jugoslawien in der ursprünglich als "slawisch" akzentuierten diplomatischen Kooperation in der Deutschlandfrage zwischen der UdSSR, der CSR und Polen hinterlassen hatte. Ende 1954 fand noch unter Teilnahme Chinas die Moskauer Konferenz der osteuropäischen Regierungen statt. Damit endete die Tradition der Stalinschen Kollektivdiplomatie, denn der Protest gegen eine atomare Bewaffnung der Bundeswehr auf der Prager Konferenz der Außenminister der ČSR, Polens und der DDR vom April 1958 befriedigte propagandistische und nicht mehr politische Zwecke. Der Schwerpunkt der internationalen Initiativen hatte sich unter Chruschtschow auf parteipolitische Foren verlagert.

Die sehr knappe Feststellung, die 1988 ein polnischer Autor zur Charakterisierung der damaligen polnischen Deutschlandpolitik benutzte, kann man auf alle ostmitteleuropäischen Länder beziehen: Ihr Inhalt sei die Unterstützung „1. der Initiativen der DDR gegenüber der Bundesrepublik und 2. der Initiativen der UdSSR gegenüber den westlichen Okkupationsmächten" auf allen Foren gewesen ${ }^{15}$. Hervorzuheben ist, daß sich alle Länder, die die DDR damals politisch unterstützten, mit ihr de jure noch im Kriegszustand befanden; der Westen legte im Verhältnis zur Bundesrepublik Deutschland einen größeren Wert auf diplomatische Distanz. Den Kriegszustand mit Deutschland beendete erst die sowjetische Erklärung vom 25. Januar 1955, der sich bis April die Satellitenstaaten anschlossen ${ }^{16}$. Hinzuweisen ist ebenfalls auf den Umstand, daß Staatsbesuche auf höchster Ebene die politische Normalisierung der bilateralen Beziehungen Berlins mit Warschau und Prag protokollarisch bekräftigten: Im Dezember 1950 reiste Pieck nach Warschau und im Oktober 1951 nach Prag, im April 1951 kam der polnische und ein Jahr später auch der tschechoslowakische Partei- und Staatschef nach Berlin. In beiden Fällen soll erheblicher Moskauer Druck nötig gewesen sein, damit Bierut und Gottwald überhaupt und auch noch rechtzeitig vor der 2. SED-Parteikonferenz nach Berlin fuhren. Diese bilateralen Staatsbesuche bildeten in Stalins Konzept der ,konzentrierten Diplomatie“ übrigens eine absolute Ausnahme, weil sie nach den Erfahrungen mit der persönlichen Diplomatie Dimitrows und Titos als obsolet galten.

15 Kowalski, Polska w swiecie, S. 444.

16 Am 3. 2. 1955 die ČSR, am 18. 2. Polen, am 1. 3. Bulgarien, am 8. 3. Rumänien, am 15. 3. Ungarn, am 17. 3. Albanien, am 7. 4. China. Da die DDR zum Zeitpunkt des Kriegsausbruchs nicht bestanden hatte und die staatsrechtliche Kontinuitätstheorie in der DDR wie im damaligen „Ostblock“ abgelehnt wurde, kann diese Erklärung nur auf das Rechtssubjekt „Deutschland als Ganzes“ bezogen werden. Wie oben vermerkt, wird dieser Rechtsakt nicht einheitlich interpretiert. 
Über die diplomatischen Anfangsschwierigkeiten klagte Anton Ackermann, damals Staatssekretär im Außenministerium, am 14. April 1950 dem Chef der sowjetischen diplomatischen Mission in der DDR. So wollten die "tschechischen Freunde“ keinen politischen Vertrag mit der DDR abschließen, sondern nur ein Handelsabkommen, der tschechoslowakische Entwurf werde deshalb dem Botschafter übergeben mit der „Bitte, bei der sowjetischen Regierung um Rat zu fragen“. Am 23. Juni 1950 wurde dann in Prag nur eine völkerrechtlich minderwertige Deklaration unterschrieben ${ }^{17}$. Ackermann berichtete dem sowjetischen Botschafter außerdem darüber, daß sich im SED-Politbüro Grotewohl über die schlechte Behandlung der DDR-Delegation in Budapest beschwert habe, die „immer auf den letzten Platz" gesetzt worden sei. Als Außenminister Dertinger (CDU) deshalb vorschlug, künftig einen Kommunisten ins Ausland zu schicken, erklärte Ackermann pikiert, daß die SED keinen Unterschied zwischen Kommunisten und Sozialdemokraten mache. Dertinger erwiderte darauf leger: „Aber das wissen sie in Prag, Warschau und Budapest nicht." 18 Man disponierte in Ost-Berlin aber um und schickte schließlich Ulbricht als Emissär nach Prag und Warschau, nachdem er über die Frage der Demarkationslinie an Oder und Neiße „ein Gespräch mit der Führung der SKK gehabt hatte"19. Dertingers Personalpolitik wurde noch in den sechziger Jahren beachtet: Bei allen in Osteuropa eingesetzten DDR-Botschaftern handelte es sich um Altkommunisten, die sich während des „Dritten Reiches“ in der politischen Emigration aufgehalten oder in Deutschland langjährige Haftstrafen verbüßt hatten. In einigen Fällen vertraten sudetendeutsche Kommunisten die DDR im „befreundeten Ausland“.

Vorab wurde der sowjetische Diplomat 1950 auch über die Details der Verhandlungen mit Prag informiert, wo man wünschte, daß die Vertreibung der Sudetendeutschen von der DDR-Regierung als gerecht, endgültig und unabänderlich anerkannt werde; außerdem legte Prag Wert auf die Ungültigkeit des Münchener Abkommens von $1938^{20}$. Im diplomatischen Hintergrund ist zu beachten, daß der Prozeß der politischen Einbindung der DDR in die sowjetische Hegemonialsphäre wegen der geopolitischen Lage blockinterne Konflikte auslöste bzw. auffrischte. Beachtet man beispielsweise, was die zwischen der DDR und der ČSR abgeschlossene Deklaration nicht enthält, und vergleicht man außerdem den polnischen und den tschechoslowakischen Text über die Beendigung des Kriegszustandes mit Deutschland, so ist der im tschechoslowakischen Wortlaut enthaltene explizite Rechtsvorbehalt hinsichtlich der Behandlung Deutschlands „als Ganzes“ unter anderem auch dahingehend zu interpretieren, daß die ČSR unter stillschweigender Berufung auf den Versailler Vertrag auf Rechten bestand, die aufgrund der nach dem Zweiten Weltkrieg veränderten territorialen Lage Polen belasteten ${ }^{21}$. Daraus resul-

17 Vgl. Gemeinsame Deklaration der Provisorischen Regierung der DDR und der Regierung der ČSR vom 23. 6. 1950, in: Dokumente zur Außenpolitik der DDR, Bd. I, S. 377-378.

18 AWP RF 82/37/6/199, Bl. 26-28, Vermerk über das Gespräch Puschkins mit Ackermann vom 14. 4. 1950.

19 Ebenda, Bl. 45-46, Vermerk über das Gespräch Puschkins mit Ackermann vom 2. 6. 1950.

20 Ebenda, Bl. 40-41, Vermerk über das Gespräch Puschkins mit Ackermann vom 19. 6. 1950.

21 Danach genoß die ČSR in Deutschland Transport- und Postprivilegien, u.a. verfügte sie auch über eine Freihandelszone im Stettiner Hafen. 
tierende polnisch-tschechoslowakische Differenzen konnten erst 1958 bereinigt werden.

Nach dem Scheitern der Stalin-Note verpflichtete Stalin Anfang April 1952 eine Delegation der SED-Führung, die DDR zum Bollwerk des volksdemokratischen Lagers gegen Westdeutschland auszubauen ${ }^{22}$. Im Hinblick auf die ideologische Integration der DDR in die sowjetische Peripherie mag der Beschluß der 2. Parteikonferenz der SED vom Juli 1952, in der DDR den Sozialismus aufzubauen, nicht unwichtig gewesen sein. Im Zentrum der Interessen Moskaus und seiner Satelliten stand jedoch der ostdeutsche Wehrbeitrag, denn die politische Emanzipation und Integration der DDR in den „Ostblock“ war das Spiegelbild entsprechender Fortschritte in Westdeutschland.

Dem Ziel der Stärkung der politischen Position der DDR diente auch eine Reihe wirtschaftspolitischer Maßnahmen der Besatzungsmacht, die in einigen Fällen durch andere Länder mitgetragen wurden. So betraf 1950 beispielsweise die Reduzierung der Reparationskosten um 50 Prozent und die Streckung der Restschulden auf 15 Jahre Polen, das zunächst mit 15 und ab März 1947 mit 7,5 Prozent am Reparationsaufkommen beteiligt war. Auch 1953 schloß sich Polen dem sowjetischen Reparationsverzicht an, was bald zu einer schweren Belastung der polnisch-sowjetischen Beziehungen führen sollte. In der politischen Klasse und 1956 auch in der Öffentlichkeit tauchte das Argument auf, daß Polen infolge der nach dem Krieg mit der UdSSR abgeschlossenen Sonderverträge über die Lieferung oberschlesischer Kohle faktisch für Deutschland (und zumindest teilweise an die DDR) Reparationen zahle.

\section{Im Zeichen der „Zwei-Staaten-Theorie“}

Die in einer ostdeutsch-polnischen Erklärung von 1955 enthaltene Floskel, wonach die Freundschaft mit der DDR - insbesondere auch im Hinblick auf „die definitive Festlegung der Oder-Neiße-Grenze“ - „hervorragende Bedeutung für den Frieden in Europa" habe, und im übrigen ein gemeinsamer Standpunkt zur friedlichen und demokratischen Vereinigung Deutschlands herrsche, könnte als Geschäftsgrundlage zumindest der aktiven Bemühungen um politische Integration der DDR betrachtet werden. Solche Phrasen wiederholen sich und lassen auf einen geschäftsmäßigen Leerlauf schließen. Aussagefähiger war schon die Formel, daß „der Aufenthalt der sowjetischen Truppen auf dem Boden der DDR eine hinreichende Garantie für die Stabilität der volksdemokratischen Ordnung in der DDR" schaffe ${ }^{23}$, die man in Moskau im Mai 1953 zur Begründung der Ablösung der militärisch dominierten Sowjetischen Kontrollkommission durch ein dem Außenministerium angeschlossenes Amt des Hohen Kommissars benutzte. Dieses Amt wurde nach zwei Jahren ebenfalls suspendiert. Als Begründung diente jetzt die Erklärung, daß „in der DDR die Beschlüsse des Kontrollrates in Deutschland [...] verwirklicht worden sind“

22 Vgl. Wettig, Abhängigkeiten und Handlungsspielräume der SBZ/DDR, S. 2595.

23 Ebenda, S. 2607. 
und damit auf dem Gebiet der DDR ihre Gültigkeit verlieren. ${ }^{24}$ Mit dem Vertrag vom 20. September 1955 übertrug die UdSSR der DDR Souveränitätsrechte ${ }^{25}$. Damit fiel auch die Kontrolle der Grenzen und der Demarkationslinie der DDR zu, Vorbehaltsrechte sicherte sich die UdSSR lediglich gegenüber Berlin und seinen militärischen Verkehrsverbindungen nach Westdeutschland. Im März 1957 folgte ein Vertrag über die zeitweilige Stationierung sowjetischer Streitkräfte auf dem Territorium der DDR. Diese hießen aber weiterhin „Gruppe der sowjetischen Streitkräfte in Deutschland“. Entlastet wurde die DDR auch von den Unterhaltskosten: 1956 wurden sie von 1,6 Milliarden auf 800 Millionen Mark im Jahr reduziert, ab 1. Januar 1959 verzichtete die UdSSR völlig darauf.

Die Schlußfolgerung, daß solche Schritte nur das Ziel verfolgten, der DDR mehr politische Verantwortung und rüstungswirtschaftliche Lasten aufzuladen, leidet unter dem Umstand, daß die DDR ab 1953/54 durch massive sowjetische Kredite gestützt werden mußte ${ }^{26}$. Dem historischen Blick entziehen sich bislang auch weitere finanzielle Transaktionen zugunsten des DDR-Staatshaushalts, etwa durch Verzicht auf sowjetische Staatskredite für SAG-Betriebe. Andererseits gehen Wirtschaftshistoriker davon aus, daß der SBZ/DDR in der Zeit von 1947 bis 1956 infolge Preisdiskriminierung im Handel mit der UdSSR geschätzte Verluste von 0,8 bis 2,0 Milliarden Mark ${ }^{27}$ entstanden sind, weil die Preise 10 bis 25 Prozent unter dem Weltmarktniveau lagen. Die danach eingefahrenen Gewinne, als die DDR für billige Rohstoffe qualitativ minderwertige Industriewaren in den sechziger Jahren zu doppelten und in den achtziger Jahren sogar zu dreifachen Weltmarktpreisen nach der UdSSR und nach Osteuropa exportierte ${ }^{28}$, fehlen in solchen ökonomischen Berechnungen. Buchheim und Herbst argumentieren, daß die Sowjetunion „für ihre politische und militärische Hegemonie im ,Ostblock' wirtschaftlich recht große Kosten“ in Kauf nahm, weil sie die DDR „seit Ende der fünfziger Jahre faktisch subventioniert hat“. Da bis zur Einstellung der Reparationszahlungen ab 1. Januar 1954 unter dem Strich wohl nicht von Subventionierung gesprochen werden kann, wird davon auszugehen sein, daß die DDR von der Sowjetunion ab diesem Zeitpunkt alimentiert wurde. Die UdSSR subventionierte die DDR nicht nur direkt, sondern akquirierte auch Beiträge von anderen RGW-Ländern, beispielsweise von Polen, worauf bereits hingewiesen wurde, oder auch von der ČSR, worauf noch eingegangen wird.

Auf politischer Ebene hatten die Resultate der beschleunigten und die Perspektiven der weiteren Integration der DDR schon früher zu Widersprüchen geführt: So war im ursprünglichen, im Moskauer Außenministerium erstellten und von Molotow präsentierten Entwurf des Warschauer Vertrages die Aufnahme der DDR (und Albaniens) gar nicht vorgesehen ${ }^{29}$. Im Hinblick auf die DDR rechtfertigte sich

24 Vgl. Beschluß der Regierung der UdSSR: Über die Auflösung der Hohen Kommission der UdSSR in Deutschland, in: Dokumente zur Außenpolitik der DDR, Bd. III, S. 284-285.

$25 \mathrm{Vgl}$. Vertrag über die Beziehungen zwischen der DDR und der UdSSR vom 20.9. 1955, in: ebenda, S. 280-283.

26 Für 1957 etwa durch Erhöhung der Warenlieferungen um 30\% gegenüber 1956 und einen Kredit von 340 Millionen Rubel in frei konvertierbarer Währung.

27 Baar/Karlsch/Matschke, Kriegsschäden, Demontagen und Reparationen, S. 946.

28 Buchheim, Wirtschaftliche Folgen, S. 359.

29 Molotow wurde als Außenminister am 1.6. 1956 durch D. T. Schepilow abgelöst. 
Außenminister Molotow im sowjetischen Parteipräsidium mit dem Argument, daß die UdSSR doch nicht wegen der DDR mit dem Westen Krieg führen würde. Chruschtschow setzte sich aber mit seiner Meinung durch, aus ideologischen Gründen alle osteuropäischen ${ }_{n}$ sozialistischen "Staaten im Bündnisvertrag zu berücksichtigen $^{30}$. Hier ist aber nicht der Ort, um die Auswirkungen des österreichischen Neutralitätsvertrages auf die Blockpolitik zu thematisieren. Gleichwohl muß die von Gerhard Wettig vorgetragene Meinung zurückgewiesen werden, daß der Buchstabe des Warschauer Vertrags die DDR aufgrund unterschiedlicher Definition der Beistandspflicht diskriminiert habe ${ }^{31}$, denn der deutsche Wortlaut weicht nicht von den anderssprachigen Vertragsfassungen $\mathrm{ab}^{32}$. Materiell privilegierte Artikel 3 des Vertrages sogar die DDR, denn er fixierte Konsultationspflicht der Vertragspartner, „wenn nach Meinung einer der Seiten die Gefahr eines bewaffneten Überfalls auf einen oder mehrere Teilnehmerstaaten des Vertrages entsteht ${ }^{\text {. }}$. Die Präambel hatte nämlich „die aggressiv-revanchistischen Kräfte" in Westdeutschland lokalisiert. Der Warschauer Vertrag verdient auch insoweit Aufmerksamkeit, weil er fast zehn Jahre vor dem Vertrag über Freundschaft, Zusammenarbeit und gegenseitigen Beistand mit der UdSSR vom 12. Juni 1964 abgeschlossen wurde. Erst dieser Vertrag leitete nämlich die definitive Einbeziehung der DDR in das zwischen 1943 und 1949 geknüpfte Netz bilateraler Beistands- und Freundschaftsverträgen der UdSSR mit ihren osteuropäischen Verbündeten ein. Die DDR kam so zwanzig Jahre später in den völkerrechtlichen Genuß „brüderlicher Zusammenarbeit“" 33 mit der UdSSR als ihre östlichen Nachbarn, die die DDR aber aufgrund ihrer exponierten geostrategischen Lage politisch schon mit dem Warschauer Vertrag abgehängt hatte. Ähnliche Freundschafts- und Beistandsverträge mit Polen, der ČSSR, mit Ungarn und Bulgarien von 1967 sowie mit der Mongolei von 1968 markierten den Abschluß der Integration der DDR in die sowjetische Peripherie. In diesem Zusammenhang ist es zumindest auffällig, daß die DDR bereits am 25. Dezember 1955 mit der Volksrepublik China und am 22. August 1958 mit der Mongolei die ersten Verträge über "Freundschaft und Zusammenarbeit" abgeschlossen hatte. Daß man Ost-Berlin in Osteuropa zehn Jahre lang die kalte Schulter gezeigt hatte, kann nicht ausgeschlossen werden. Aber auch darüber, wie Ulbricht in der zweiten Hälfte der fünfziger Jahre die „chinesische Karte“ zu spielen gedachte, weiß man nur sehr wenig. Aufschlußreich ist überdies ein Blick in die Vertragstexte von 1967: Im Vertrag mit Polen verpflichtete sich die DDR ausdrücklich zur Verteidigung der Oder-NeißeGrenze, die sie bereits 1950 anerkannt hatte; im Vertrag mit der ČSSR wurde das Münchener Abkommen formell von Anfang an für nichtig erklärt. Nur lag zu diesem Zeitpunkt diese (gegenüber der Bundesregierung aus völker- wie verfassungs-

$30 \mathrm{Vgl}$. Zentralno-Wostotschnaja Ewropa, Bd. I, S. $272 \mathrm{f}$.

31 Laut Wettig, Abhängigkeiten und Handlungsspielräume der SBZ/DDR, S. 2620 wurde in der deutschen Übersetzung des Vertragstextes der Umfang der Beistandspflicht nach Artikel 4 nicht von dem Vertragspartner selbst, also von der DDR, wie es sonst im russischen Original und den Vertragstexten der übrigen Partner hieß, sondern umgekehrt von den begünstigten Verbündeten bestimmt.

32 Der deutsche, russische, tschechische und polnische Wortlaut des Warschauer Vertrages sind abgedruckt in: Gesetzblatt der DDR, Teil I, Nr. 46, S. 381-391.

33 Vgl. Präambel des Vertrages über Freundschaft, Zusammenarbeit und gegenseitigen Beistand zwischen der DDR und der UdSSR vom 12. 6. 1964, in: Dokumente zur Außenpolitik der DDR 1964, Bd. XII, S. 1022. 
rechtlichen sowie politischen Gründen nicht durchsetzbare) Nichtigkeitserklärung ex tunc nicht mehr im Interesse der ČSSR und blockierte lediglich die damals von Prag mit Vehemenz angestrebte Erweiterung der Beziehungen zur Bundesrepublik Deutschland. Die DDR zementierte damit ihre Vetoposition in der brüchig werdenden Deutschlanddiplomatie des nach Westen strebenden "Ostblocks“.

Doch zurück in die fünfziger Jahre: Auf dem XX. Parteitag der KPdSU revidierte Chruschtschow die sowjetische außenpolitische Doktrin, als er die von Stalin 1952 geäußerten Zweifel an seinem eigenen Dogma von der Unausweichlichkeit des Krieges aufgriff und in der „friedlichen Koexistenz“ der Systeme den „Friedenskampf" nicht mehr wie sein Lehrmeister nur im Sinne eines konfrontativen „Kampfes für die Weltrevolution" akzentuierte, sondern als eine kooperative Systemkonkurrenz in den Vordergrund stellte. Noch versöhnlichere Töne schlug Chruschtschow 1959 auf dem XXI. Parteitag an, als er feststellte, daß „die Sowjetunion nicht mehr durch Feinde umzingelt" sei und das Parteiprogramm von 1961 sogar von der Möglichkeit sprach, den Krieg überhaupt beseitigen zu können. In diesem Konzept der "friedlichen Konkurrenz" sollte die DDR als „Frontstaat“ die Bundesrepublik in wichtigen Konsumpositionen überholen. 1958 setzte Ulbricht auf dem V. SEDParteitag öffentlich zum "Großen Sprung“ an und erklärte zur „ökonomischen Hauptaufgabe", den westdeutschen Pro-Kopf-Verbrauch bei wichtigen Konsumgütern bis 1961 einzuholen ${ }^{34}$, ein Jahr später verlängerte er die Frist bis $1965^{35}$. Diese ostdeutschen und sowjetischen Zielvorgaben sollte die DDR mit Unterstützung der RGW-Partner erreichen. 1960 endete Ulbrichts "Großer Sprung" mit einer Bauchlandung: In der DDR mußten einige Grundnahrungsmittel rationiert werden. Verstärkte Abhängigkeit von der UdSSR und dem RGW war die Folge.

Tatsächlich hatte die SED damals aber auch die KPdSU schon weit hinter sich gelassen, denn das Ziel, die „Versorgung der Bevölkerung, besonders der Arbeiterklasse, mit Industriewaren sowie Nahrungs- und Genußmitteln über den Stand von Westdeutschland zu heben“ und einen "sichtbar höheren Lebensstandard in der DDR als in Westdeutschland" zu erreichen, gehörte schon zu den Aufgaben des „Neuen Kurses“. Intern war sie 1954 als Verhandlungsbasis der SED für RGW-Verhandlungen fixiert worden ${ }^{36}$ und im Januar 1956 forderte Ulbricht den Ausbau des $\mathrm{RGW}$ „zu einem zentralen Organ [...], das in Zusammenarbeit mit Gosplan der UdSSR die großen Aufgaben der Volkswirtschaft in den volksdemokratischen Ländern richtungsweisend lenkt ${ }^{\text {" }} 3$. In keinem anderen Land bediente und beförderte das Eigeninteresse der herrschenden Monopolpartei unmittelbar das Kontrollinteresse der Hegemonialmacht.

Ralph Sowart bewertet die wirtschaftlichen Ergebnisse der Ulbrichtschen Überholpolitik im Nachhinein als „bescheiden“. Weniger zurückhaltend im Urteil waren die Zeitzeugen, die bereits im Planungsstadium von Hochstapelei sprachen. Der sowjetische Botschafter Puschkin schickte Außenminister Molotow am 28. Dezember 1954 einen von Wirtschafts- und Planungsfachleuten der Botschaft erstellten Be-

34 Vgl. Sowart, Planwirtschaft, S. 157-190.

35 Vgl. Staritz, Geschichte der DDR, S. 174-176.

$36 \mathrm{Vgl}$. SAPMO, NY 4090/470, Entwurf/Thesen für das Auftreten der Deleg[ation]/Berlin, den 1.3.1954.

37 Vgl. Thme-Tuchel, Das „nördliche Dreieck“, S. $121 \mathrm{f}$. 
richt, in dem stand, daß die DDR zwar hohe Jahreswirtschaftswachstumsraten verzeichne, aber dennoch unter den Zielen des Fünfjahrplans geblieben sei. Der Export sei 1954 um 36 Prozent gewachsen, aber infolge des Verzichts auf Reparationen; der Import wuchs gegenüber 1953 um 7,8 Prozent, aber aufgrund eines sowjetischen Kredits; gegenüber der UdSSR seien 1954 zehn Prozent der vereinbarten Lieferungen nicht geleistet worden. Irreal sei auch der Plan für 1955. So solle der Export nach kapitalistischen Ländern um 46 Prozent und nach den Volksdemokratien um elf Prozent wachsen. Dabei betrage der DDR-Außenhandelssaldo zum 31. Dezember 1954 minus 270 Millionen Rubel, davon 220 Millionen zugunsten der Volksdemokratien; der Realsaldo belaufe sich auf mindestens 550 bis 600 Millionen Rubel, weil die „realen Möglichkeiten und Importbedürfnisse der volksdemokratischen Länder nicht beachtet" wurden. 1954 habe die DDR 20 Prozent ihres Getreide- und 32 Prozent des Butterverbrauchs importieren müssen, bei Fleisch und Fett lagen die Anteile bei über zehn Prozent ${ }^{38}$.

Auf den Punkt brachte Ulbrichts Ehrgeiz der tschechoslowakische Staats- und Parteichef Antonin Novotny, als er in seinem Politbüro dafür plädierte, im (innerhalb des "Lagers" harmonisierten) Programm der KPTsch nur das Einholen, nicht aber das Überholen zu fixieren, „damit der Westen unseren nackten Hintern nicht sieht "39. Die ČSR, damals das einzige Land im „Ostblock", dessen Lebensstandard fast zwei Jahrzehnte mit dem Niveau westlicher Industrieländer Schritt halten konnte, wurde damals von Österreich überholt, das in der informellen "öffentlichen" Meinung als Richtschnur diente. Zu Beginn der sechziger Jahre brachen in Prag wilde Streiks aus. Flugblätter tauchten auf mit dem Text: „Wir wollen für uns arbeiten, nicht für Ulbricht." Den Hintergrund gaben Gerüchte ab, daß die UdSSR einen Teil der Kredite, die sie der DDR gewährt hatte, auf die ČSR abgewälzt hätte. Das beiderseitige Verhältnis war zu Beginn der sechziger Jahre genauso nachhaltig gestört wie das ostdeutsch-polnische nach 1956.

Größer erscheinen Ulbrichts „bescheidene“ Erfolge aus der gesamtdeutschen Perspektive, sobald man sich Details des Potsdamer Protokolls bezüglich der Deckelung des Lebensstandards in Deutschland vergegenwärtigt. Die ideologische Salvierung der ostdeutschen Überholziele durch die UdSSR und die Länder des "Ostblocks" kam nämlich einer einvernehmlichen Revision dieser Bestimmungen gleich. Hier öffnet sich eine verdrängte Perspektive der deutsch-deutschen Doppelgeschichte.

Auf politischem Gebiet lassen sich die Ergebnisse der regen Reisediplomatie zwischen den osteuropäischen Hauptstädten und Berlin im Jahr 1957 in den Schlagworten „Bestandsgarantie für die volksdemokratische Ordnung in der DDR“, Erhöhung der Warenlieferungen und Kredite für die DDR zusammenfassen. Auf Leerlauf in der ökonomischen Sphäre deutet auch das 1958 stattfindende Expertengespräch zwischen Polen, der ČSR und der DDR über den Bau eines Oder-DonauKanals hin, denn die Vision von einer mitteleuropäischen Wirtschaftsregion diente schon seit vielen Jahrzehnten als Trostpflaster und lenkte wohl von der Inaktivität des RGW ab, der nach Beschlüssen von 1962 im Sinne einer ,internationalen sozialistischen Arbeitsteilung" ausgebaut werden sollte. Von realer Tragweite war die

38 Vgl. AWP RF 82/43/305/34, Bericht über den Wirtschaftsplan der DDR für 1955.

39 Persönliche Mitteilung von Zdenek Mlynar. 
1958 gefallene Entscheidung, von der UdSSR eine Fernleitung für Erdöl und Erdgas nach Ungarn, Polen, die DDR und die ČSR zu legen. Die DDR bekam 1973 einen Anschluß, 1975 wurde die Erdgasleitung nach der Bundesrepublik verlängert.

Der Warschauer Pakt hatte in den fünfziger Jahren als Integrationsfaktor keine praktische Bedeutung: Im Mai 1958 bestätigte in Moskau der Politische Beratungsausschuß die Aufnahme von NVA-Truppen in das Vereinte Kommando. Auf der Beratung nahmen der polnische und der tschechoslowakische Außenminister im Sinne der Entspannungspolitik einen mäßigenden Einfluß auf den Stil der Deklaration. Polnischerseits wurde eine einzige Verschärfung bezüglich des Verhältnisses zur Bundesrepublik Deutschland erzielt, die von tschechoslowakischer Seite unterstützt wurde ${ }^{40}$. Ein DDR-Teilnehmer wurde im (bisher unvollständig zugänglichen) Bericht nicht erwähnt, und generell analysierte man in Prag den Grundtenor dahingehend, daß die UdSSR vor allem an einer Normalisierung ihrer Beziehungen zur Bundesrepublik Deutschland interessiert sei. Auf dem Folgetreffen im Februar 1960 zogen die ostdeutsche, die polnische und die tschechoslowakische Delegation an einem Strang. Gemeinsam erreichten sie eine Verschärfung der diplomatischen Verbalattacken auf Westdeutschland. Der im Entwurf vorgesehene Passus „volles Verständnis für Aktionen der DDR zur Minderung der internationalen Spannung“" wurde auf Vorschlag der Prager Vertreter in „volle Unterstützung der Aktionen“ geändert ${ }^{41}$. Wenn man sich aber bemüht, die hinter den öffentlichen Verlautbarungen stehende Absicht zu erforschen, fällt außer der polnischen Abstinenz auf, daß in internen Gesprächen zwischen sowjetischen und tschechoslowakischen Diplomaten der sowjetischen Seite durchaus bewußt war, daß die in Berlin nach Kontrollratsrecht bestehenden (und im Westteil der Stadt angesiedelten) Militärmissionen einiger Staaten des Warschauer Paktes der öffentlich gemeinsam festgelegten Behandlung der deutschen Hauptstadt als selbständige politische Einheit zwar im Wege standen, ihre Umwandlung in Konsulate jedoch für aussichtslos gehalten wurde ${ }^{42}$. Trotz aller politischen und diplomatischen Spannungen hatten nicht nur Moskau und Prag die Normalisierung der Ost-West-Beziehungen als Ziel vor Augen. Zwischen der DDR und der ČSSR sollten um den Berlin-Status bald heftige diplomatische Konflikte ausbrechen. Ihnen folgten öffentliche Polemiken, die erst mit der Intervention des Warschauer Paktes in der Tschechoslowakei 1968 aufhörten.

Wenn man den Bau der Berliner Mauer als eine Zäsur im Prozeß der Integration der DDR in den „Ostblock“ sehen möchte, dann muß unbedingt darauf hingewiesen werden, daß über das Verhältnis zwischen der DDR und der UdSSR am Ende der fünfziger Jahre einige Forschungskontroversen die Orientierung erschweren. Umstritten ist der Initiator der Mauer: Chruschtschow oder Ulbricht. Manche hal-

40 1958, 7. cerven, Praha. - Informace o porade Politickeho poradniho vyboru Varsavske smlouvy dne 24. 5. 1958, predlozena politickemu byru UV KSC ministrem zahranici ČSR V. Davidem, in: Reiman/Lunak, Studena valka, S. 77-81, hier S. 79.

41 1960, 20. unor, Praha. - Zprava ministra zahranici V. Davida o zasedani Politickeho poradniho vyboru Varsavske smlouvy v Moskve dne 4. unora 1960, predlozena politickemu byru UV K.SC, in: ebenda, S. 141-147, hier S. 145.

42 1960, 20. unor, Praha. - Zprava ministra zahranici V. Davida o zasedani Politickeho poradniho vyboru Varsavske smlouvy v Moskve dne 4. unora 1960, predlozena politickemu byru UV KSC, in: ebenda, S. 141-147, hier S. 145. 
ten Ulbricht für den Treibenden und Chruschtschow für den Getriebenen (Hope Harrison), anderer Meinung ist Michael Lemke ${ }^{43}$, der diese Entscheidung vor allem auf die wirtschaftlichen Schwierigkeiten der DDR zurückführt, die die Abhängigkeit von der UdSSR erhöhten. Wertet man das von Bonwetsch und Filitow veröffentlichte Protokoll des von Ulbricht beantragten Moskauer Gipfeltreffens der Warschauer-Pakt-Staaten in der ersten August-Woche $1961^{44}$ aus und zieht außerdem andere Quellen zu Rate ${ }^{45}$, um aus dem Originalton und den Auslassungen weitere Hinweise zu entnehmen, gewinnt man ein sehr farbiges Bild. Der SED-Chef forderte von den osteuropäischen Staaten unumwunden die vertragliche "Gewährleistung der wirtschaftlichen Unabhängigkeit der DDR von der Bundesrepublik“ und vom RGW „die kontinuierliche Versorgung der Bevölkerung der DDR im IV. Quartal 1961 und im Jahre 1962“. Gleichzeitig kündigte er an, daß die DDR ihre Lieferverpflichtungen nicht erfüllen könne. Gomulka, der als erster antwortete, stellte ebenso direkt fest, daß die Lage in der DDR eine Grenzschließung nach Westberlin erforderlich mache und außerdem eine Umlenkung der innerdeutschen Handelsströme der DDR nach Osten ${ }^{46}$. Novotny zeigte sich einleitend verbal hilfsbereit, aber konkret könne die ČSSR nicht mit landwirtschaftlichen Produkten helfen, weil sie nicht einmal ihren eigenen Bedarf decken könne. Auch sehe er sich nicht in der Lage, in die DDR Güter zu liefern, die die ČSSR selbst aus der Bundesrepublik importieren müsse ${ }^{47}$. Die wenig diskreten Vorhaltungen Gomulkas und Novotnys setzte in seiner Abschlußrede Chruschtschow fort. Der DDR wurde nur politische Hilfe zugesichert.

\section{Zusammenfassung}

1. Die sowjetische Hegemonie über Ostmitteleuropa basierte auf militärischer Grundlage. Der Warschauer Pakt garantierte der sowjetischen Armee jene Bewegungsfreiheit, die ihr im Friedensvertrag von 1947 nur für die Dauer der Besetzung Österreichs eingeräumt worden war. In diesem System nahm die SBZ/DDR als besatzungsrechtliches Kondominium bis 1954/55 und danach als der größte sowjetische Militärstützpunkt in Ostmitteleuropa, der gleichzeitig Polen und die ČSR als militärisches Transit- und Hinterland legitimierte, geopolitische Ordnungsfunktionen wahr. Diese wurden optimiert durch das Junktim zwischen der „deutschen Frage" und dem globalen Ost-West-Konflikt. Die Verleihung von Attributen staatlicher Souveränität an die DDR hing überhaupt einerseits ursächlich mit deren Integration in die Struktur des sowjetischen Hegemonialbereichs zusammen und beschleunigte darin andererseits alle Integrationsprozesse. Faktisch entsprach der Grad der Blockintegration der DDR jeweils dem Grad der Unterordnung der übrigen Länder unter die Interessen der Hegemonialmacht. Dieser Strukturkonflikt potenzierte sich durch jede aktuelle Identität zwischen sowjetischen und ostdeutschen

43 Vgl. Lemke, Berlinkrise.

44 Bonwetsch/Filitow, Chruschtschow und der Mauerbau, S. 155-198.

45 Reiman/Lunak, Studena valka, S. 308-313.

46 Bonwetsch/Filitow, Chruschtschow und der Mauerbau, S. $174 \mathrm{f}$.

47 Ebenda, S. 178. 
Interessen, weil er der SED erlaubte, ihre politischen Sonderinteressen als politische Gesamtinteressen des „Ostblocks“ und umgekehrt darzustellen. Konstant war ab 1953 im „Ostblock“ nur das Interesse an der Stabilität der SED-Herrschaft, die in der gegebenen politischen Lage nicht mehr, wie in der vorangegangenen Stalinschen Phase, ausschließlich mit der abstrakten ideologischen Identifikation begründet wurde, sondern zunehmend auch schon sicherheitspolitisch. Oder einfacher gesagt: Der eigentliche Maßstab für die Ostintegration der DDR war die sowjetisch-osteuropäische Wahrnehmung Westdeutschlands.

2. Der „Ostblock“ war aber nicht nur Ergebnis ideologisch, militärisch, ökonomisch und technologisch motivierter politischer Zwangsintegration durch die UdSSR, sondern unter den gegebenen globalpolitischen Rahmenbedingungen auch immanente Folgewirkung der in den betroffenen Ländern nach 1945 insbesondere auf wirtschaftlichem Gebiet eingeschlagenen Innenpolitik. Die ältere Schlußfolgerung, daß die UdSSR sich „mit dem RGW [...] ein Instrument [schuf], das es unverzüglich und zuverlässig ermöglichen sollte, die traditionellen Handelsbindungen der Länder Ostmittel- und Südosteuropas und schließlich im Jahre 1950 auch der DDR an den Westen institutionell und funktionell umzulenken und sie binnen- und außenwirtschaftlich den eigenen Zielen unterzuordnen ${ }^{* 48}$, trifft auch für die DDR nur bedingt zu, denn der SBZ-Export (ohne innerdeutschen Handel) war schon 1946 zu 99 Prozent nach späteren RGW-Ländern orientiert ${ }^{49}$. Zunächst vollständig durch die Besatzungsmächte dirigiert und nach 1948 durch GATT und OEEC vom Westen isoliert, wickelte die DDR 1950 schon 47,3 Prozent ihres Außenhandels mit der UdSSR $\mathrm{ab}^{50}$ (nach der veröffentlichten DDR-Außenhandelsstatistik nur 39,7 bzw. 40 Prozent nach internen statistischen Angaben ${ }^{51}$ ). 1952 betrug der Anteil ihres Außenhandels mit der Sowjetunion und den Volksdemokratien 78 Prozent (nach offiziellen Angaben nur 72, nach „internen“ Daten 73,7752 bzw. 68,2 Prozent nach „neuer“ Berechnung ${ }^{53}$ ). Unter Zugrundelegung der amtlichen DDR-Statistik war die wirtschaftliche Abhängigkeit der DDR vom blockinternen Außenhandel Mitte der fünfziger Jahre weit stärker und im Hinblick auf den wirtschaftlichen Austausch mit der UdSSR doppelt so stark ausgeprägt wie bei ihren osteuropäischen Kooperationspartnern ${ }^{54}$.

1960 hat sich der Außenhandelsumsatz der DDR gegenüber 1950 verfünffacht ${ }^{55}$. Überproportional expandierte nur der Handel mit der UdSSR, der Westhandel blieb unter dieser Wachstumsquote. War die DDR 1950 noch mit 10,7 Prozent am gesamten Außenhandelsumsatz des RGW beteiligt, so stieg der Anteil 1960 auf

48 Schüller/Hamel, Integration der DDR-Wirtschaft, S. 2700.

49 So laut Schwarzer, Sozialistische Zentralplanwirtschaft, S. 18. Anders: Buchheim, Wirtschaftliche Folgen, S. 348 und S. 350. Danach betrug der Anteil Osteuropas am SBZ-Außenhandelsumsatz (einschließlich des innerdeutschen Handels) $19478 \%, 1948$ 47\%, $194956 \%$.

50 AWP RF 082/40/5/254, Bl. 21-39, Puschkin/Torgowyje swjazi SSSR s GDR w 1951 g./28. 3. 1952.

51 Vgl. Buchheim, Wirtschaftliche Folgen, S. 351.

52 Ebenda, S. 351.

53 Laut Statistischem Jahrbuch von 1990, zit. nach Ahrens, Gegenseitige Wirtschaftshilfe, S. 62.

54 Umsatzabhängigkeit vom RGW-Handel im Vergleich: Ungarn 61\%, Polen 64\%, ČSR 66\%; der Anteil der UdSSR am Außenhandel betrug 1956 bei der DDR über 40\%, bei Ungarn $25 \%$, bei Polen $27 \%$ und bei der ČSR über 30\%\% Vgl. Engert, Lexikon des RGW, S. 276.

55 Vgl. ebenda, S. 280. 
16,656. Damit befand sich die DDR im Verein mit Bulgarien und der UdSSR, denn bei allen anderen osteuropäischen Ländern war in dieser Zeit der RGW-Handel anteilsmäßig zurückgegangen ${ }^{57}$. Berücksichtigt man dabei, daß 1950 auch die UdSSR 80 Prozent ihres Außenhandelsumsatzes mit RGW-Ländern abgewickelt hat, dann waren die UdSSR und die DDR in gleich hohem Maß vom "geschlossenen Wirtschaftsmarkt" des „Ostblocks" abhängig und faktisch auf ihn angewiesen.

Das Bild von der Abhängigkeit der DDR vom Außenhandel mit der UdSSR wird noch konkreter, wenn man beachtet, daß 1952 über ein Drittel des sowjetischen Exports in die DDR Lebensmittel und fast 50 Prozent Rohstoffe ausmachten ${ }^{58}$. Allein 1953 kamen aus der Sowjetunion auf der Grundlage des gewöhnlichen Handelsvertrags 935000 Tonnen Getreide ${ }^{59}$, das waren 90 Prozent des gesamten Getreideimports und fast die Hälfte des in der DDR statistisch ausgewiesenen Pro-Kopf-Jahresverbrauchs ${ }^{60}$. Die DDR exportierte in die UdSSR zu über 50 Prozent Maschinen und Ausrüstungen. Allein der geringe Investitionsaufwand der DDR für den UdSSR-Export von nur 1,26 Milliarden Mark im Zeitraum von 1948 bis 1965 - das waren nur zwei Prozent der gesamten Investitionssumme ${ }^{61}$ - erscheint geeignet, um im Zusammenhang mit der wirtschaftlichen und politischen Integration der DDR in den "Ostblock" außer machtpolitischen und ideologischen Aspekten auch solche zu beachten, die auf der DDR-Seite unmittelbar Probleme auf den Gebieten Arbeits- und Absatzmarktpolitik oder Kapitalmangel betrafen, und die auf der Seite ihrer Integrationspartner als unmittelbare oder - insbesondere im Bereich der technologischen Modernisierung - mittelbare Kosten thematisiert werden müssen.

3. Die DDR war nicht als ein „Brückenstaat“ zwischen West und Ost, sondern als ein „Sperrstaat" entstanden. Politisch sperrte sie den Westen von Osteuropa ab, eine Funktion, die in den ursprünglichen Planungen Stalins Polen gegenüber Deutschland zugedacht war. Aus binnenwirtschaftlichen wie innenpolitischen Gründen schnitt die DDR aber auch Ostmitteleuropa vom Westen wirtschaftlich, technologisch und kulturell ab.

Als Instrument der sowjetischen Kontrolle über Mitteleuropa war die DDR auf die allseitige politische und wirtschaftliche Protektion und Alimentierung durch die Hegemonialmacht angewiesen. Die politischen Folgekosten waren für die UdSSR und ihren gesamten Hegemonialbereich letzten Endes ruinös. Dennoch erfolgte die formelle Gleichstellung der DDR mit anderen Ländern stufenweise und vergleichs-

56 Ebenda, S. 281.

57 Der DDR-Außenhandel war 1960 gegenüber 1950 um das Fünffache gewachsen, mit der UdSSR um das 5,4-, mit den RGW-Ländern um das 4,7fache und fast um das Vierfache mit sogenannten entwickelten kapitalistischen Staaten. Vgl. Statistisches Jahrbuch DDR 1973, S. 33.

58 AWP RF 082/41/57/276, Bl. 8, Torpredstwo SSSR w GDR/Ottschot o rabote Torpredstwa SSSR w GDR sa $1952 \mathrm{god} / 7.2 .1953$.

591953 wurden Lebensmittel für 1130 Millionen Rubel eingeführt, d.i. 280 Millionen US-Dollar. Vgl. ADN 20. 7. 1953, in: Dokumente zur Außenpolitik der DDR, Bd. I., S. 274-275.

60 Statistisches Jahrbuch DDR 1958, S. 576 sowie S. 28 f. im Anhang III. Darin wurde der gesamte Ernteertrag mit $5699000 \mathrm{t}$ angegeben, 1955 wurden pro Kopf $118,4 \mathrm{~kg}$ Getreideerzeugnisse verbraucht, was bei einer Bevölkerung von 18 Millionen $2131000 \mathrm{t}$ ausmacht. Ebenda, S. 237.

61 Bei einer Gesamtinvestionssumme von ca. 60 Milliarden Mark im Zeitraum 1956-1964. Vgl. Ahrens, Gegenseitige Wirtschaftshilfe, S. 105. 
weise spät: Erst ab 1959 verzichtete etwa die UdSSR auf Unterhaltskosten für ihre in der DDR stationierten Truppe, und die Aufnahme der DDR in das Netz bilateraler Beistands- und Freundschaftsverträge erfolgte erst Mitte der sechziger Jahre.

Läßt man global- und deutschlandpolitische Zusammenhänge außer acht, so stellt sich die Ideologie als herausragendes Instrument dieser Alimentierungspolitik dar. Auf diesem Gebiet sind deutliche Unterschiede zwischen der Politik Stalins und Chruschtschows festzustellen. Der erste bremste die ideologisch legitimierten Beschleunigungsbemühungen in der Politik der SED mehrfach ab. So hielt er sie im Dezember 1948 etwa für „noch nicht [...] genügend reif“, um in das KominformBüro aufgenommen zu werden ${ }^{62}$. Die anfängliche Zurücksetzung der DDR fällt auch in offiziellen Deklarationen von 1951/52 auf: Alle Seiten machten damals einen klaren Unterschied zwischen dem volksdemokratischen bzw. dem sozialistischen Weg der ČSR und Polens und der antifaschistisch-demokratischen Ordnung der DDR ${ }^{63}$. Die Rhetorik von der Gemeinsamkeit des Ziels des „planmäßigen Aufbaus der Grundlagen des Sozialismus" setzte sich in der Öffentlichkeit erst ab 1955 unter Chruschtschow allmählich durch ${ }^{64}$.

Ideologische Integration war für die SED aber nicht nur doktrinäre Buchstabengläubigkeit, sondern ein effizientes Instrument der Innen- und Außenpolitik. Schon 1950 bat die SED beispielsweise um Entsendung sowjetischer Berater. Dies geschah ohne reale Not, denn die DDR wurde unmittelbar durch sowjetische Fachleute nach „sowjetischem Vorbild“ aufgebaut, und in der SKK sowie in den SAG-Betrieben wirkten damals zahlreiche sowjetische Experten und Kontrolleure. Tatsächlich wurden dann erst im Mai 1952 sowjetische Spezialisten in die DDR geschickt. Als die SED in der zweiten Hälfte 1954 in Moskau um 65 sowjetische Berater und Experten bat, genehmigte der sowjetische Apparat nur 2765. Welchen Integrationsdruck die DDR mit ihrer Vehemenz erzeugen konnte, indiziert der Umstand, daß eine gegen Slansky erhobene „schwere Anschuldigung" lautete, daß er als Generalsekretär der KPTsch nicht um Entsendung sowjetischer Berater in die ČSR bat. Erst nach der Verhaftung Slanskys schickte nämlich der Ministerpräsident der ČSR im Februar 1952 gleich sechs Bittbriefe nach Moskau, in denen er zwecks „Umbaus“ der Wirtschaft, der Justiz, der Finanzverwaltung und der Rüstungsindustrie nach „sowjetischem Vorbild“ um Delegierung sowjetischer Berater in die ČSR bat ${ }^{66}$.

Einen ähnlichen Verdrängungswettbewerb schien die SED auch auf Moskauer Parteihochschulen betrieben zu haben. Nach vorläufigen Zahlen haben 1952 und 195340 SED-Mitglieder Einjahreskurse an der Parteihochschule des ZK der KPdSU beendet, Mitte 1954 studierten in Moskau 29 SED-Mitglieder auf der Parteihochschule, und 20 weitere absolvierten dort Einjahreskurse. Für das Schuljahr 1954 bat Ulbricht, 20 SED-Mitglieder auf Jahres- und weitere zehn auf Dreijahres-

62 Friedrich, Kominform und SED, S. $332 \mathrm{f}$.

$63 \mathrm{Vgl}$. Ansprache Piecks in Warschau am 22. 4. 1951, in: Dokumente zur Außenpolitik der DDR, Bd. I, S. 360, sowie Ansprache Piecks am 11.3. 1952 beim Staatsbesuch Gottwalds in Berlin, ebenda, S. 399.

64 Initiativ in diesem Zusammenhang war Suslows Rede vom 6. 11. 1955 in Berlin, in: Dokumente zur Außenpolitik der DDR, Bd. III, S. 314.

$65 \mathrm{Vgl}$. AWP RF 82/43/305/34, Bl. 62-250, Ekonomitscheskoje poloschenije w GDR [1955].

$66 \mathrm{Vgl}$. Zapotocky/Schiroky an Regierung der UdSSR,12. 2. 1952, in: Wolokotina, Wostotschnaja Ewropa, Bd. II, S. 688-689. 
kurse schicken zu dürfen 67; Schirdewan erhöhte das Kontingent für das Schuljahr 1954/55 um weitere fünf Studienplätze an der Parteihochschule, zehn Einjahreskurse und fünf Plätze an der Akademie für Gesellschaftswissenschaften beim $Z^{6}{ }^{68}$. Erst die für die Jahre 1955/56 von der SED beantragten Studienplätze sind mit entsprechenden Zahlen der KPTsch vergleichbar: Im September 1955 beantragte die SED 20 und die KPTsch 13 Studienplätze auf der Parteihochschule des ZK der KPdSU, außerdem jeweils 20 Einjahreskurs-Plätze sowie die SED sieben und die KPTsch drei Plätze auf der Akademie für Gesellschaftswissenschaften ${ }^{69}$. Im nächsten Jahr schickte Ulbricht 18 SED-Genossen auf die einjährigen Kurse der Parteihochschule (die KPTsch nur 13), 22 auf die Parteihochschule (die KPTsch offenbar keine) und fünf zum Studium auf die Akademie für Gesellschaftswissenschaften beim ZK der KPdSU, wobei hier die KPTsch mit ebenfalls fünf mitzog 70 .

Die Ereignisse des Jahres 1956 in Polen und Ungarn begünstigten die Prestigebildung der SED auf ideologischem Gebiet genauso wie 1948 der Stalin-Tito-Konflikt. Nachdem Polen öffentlich und Ungarn heimlich aus dem „Ostblock“ ausscherten, unterhielt in der zweiten Hälfte der fünfziger Jahre die SED zusammen mit der KPTsch eine Intensivstation des Dogmatismus. Aufgrund ihrer außenpolitischen Stabilisierungsfunktion und des permanenten innenpolitischen Legitimationsdefizits fiel es der SED zugleich außerordentlich schwer, sich nach dem Ausgleich zwischen Moskau und Belgrad ideologische Freiheiten herauszunehmen. Zeitweilige maoistische Anleihen verdienen deshalb aus zwei Gründen größere Aufmerksamkeit: Erstens versuchten ab Mitte der fünfziger Jahre mehrere osteuropäische Länder die "chinesische Karte" zu spielen, zweitens deuten sie auf latente Konfliktbereitschaft der SED-Führung gegenüber der Hegemonialmacht und insgesamt auf ein funktionelleres Verhältnis zur UdSSR hin als der „erste Schein“ des schriftlichen Quellenniederschlags vermuten läßt. Ob schließlich die Ablehnung, auf die Sondierungsversuche späterer tschechoslowakischer Wirtschaftsreformer stießen, die Ende der fünfziger Jahre in der SED für ein gemeinsames Vorgehen auf diesem Gebiet geworben hatten, tatsächlich nur auf die bedingungslose Vasallentreue Ostberlins zurückzuführen war, wie tschechische und slowakische Ökonomen resigniert berichteten, oder aber nur den eigenen „Sonderweg“ der späteren NÖS-Reform schützen sollte, steht ebenfalls noch offen.

4. Die Protektions- und Alimentierungspolitik gegenüber der DDR war für andere Länder mit hohen Kosten verbunden. Im Grundsatz verschaffte sich die DDR politische Handlungsfreiheit, indem sie die Blockinteressen der Hegemonialmacht bediente. Die Wahrnehmung dieser Sicherungsfunktion war mit der Gefahr verbunden, daß die DDR bei der Gestaltung der Deutschlandpolitik des „Ostblocks“ Mitwirkungsrechte gewann. Ein Vetorecht der SED-Führung zeichnete sich $1958 \mathrm{ab}$, als der sowjetische Botschafter Puschkin faktisch zur persona non grata erklärt

67 Vgl. RGANI, Rolik 496/Delo 1082, Bl. 46, Sprawka vom 15.6. 1954.

68 Vgl. ebenda, Rolik 498/Delo 1093, Bl. 142, Vermerk vom 7. 7. 1954.

69 Vgl. ebenda, kartotek GDR 1952-1956gg. Protokoll No. 78 der Sitzung des Sekretatiats des ZK der KPdSU vom 30. 5. 1955, TOP $150 \mathrm{~g}$.

$70 \mathrm{Vgl}$. ebenda, Protokoll No. 8 der Sitzung des Sekretariats des ZK der KPdSU vom 23. 4. 1956, TOP 234g. Die Zahlen zur KPTsch stammen aus den Protokollen Nr. 19 vom 31.7. 1956, TOP 85g. und 69g., Nr. 21 vom 23. 8. 1956, TOP 33g, Nr. 80 vom 14. 7. 1955, TOP 367g., und Nr. 79 vom 11. 6. 1955, TOP 301g. Alle in: ebenda. 
wurde oder im Zusammenhang mit Protesten Ostberlins gegen West-Berlin-Reisen sowjetischer Delegierter ${ }^{71} .1958$ sei man in Moskau hellhörig geworden, als sich in der Deutschlandpolitik eine rhetorische Annäherung zwischen der DDR, Polen und der ČSR einstellte ${ }^{72}$. Ob das Berlin-Ultimatum 1958 nur Chruschtschows Faustschlag auf den Tisch war, um die sowjetische Autorität wenigstens in der Propaganda wieder herzustellen, und der Mauerbau nur den heimlichen Wünschen Gomulkas, Novotnys und Ulbrichts entsprach, bleibt abzuwarten.

Stalin definierte das spezifische Selbsterhaltungsinteresse der SED zwar als allgemeines Blockinteresse, doch als ein regional begrenztes. Prag und Warschau registrierten zu Beginn der fünfziger Jahre sehr aufmerksam Positions- und Prestigeverluste, die sie im Wettlauf mit der DDR in Moskau erlitten. Die Konflikte zwischen nationalen Interessenlagen konnten innerhalb der nach Stalins Tod instabilen Struktur des „Ostblocks“ nicht ausgetragen werden, konkrete Interessengegensätze mußten durch Bedrohungsprojektion oder durch als ideologische Fiktion getarntes Machterhaltungsinteresse gekittet werden. Konflikte, latente und offene, waren Folgen dieses durch Ideologie legitimierten und geprägten Integrationsmusters, das sich überdies in den fünfziger Jahren noch im Widerspruch zu den in Osteuropa offiziell gesteuerten, auf „Deutschland als Ganzes“ bezogenen politisch-kulturellen Wahrnehmungsstereotypen befand.

Im Grundsatz gilt aber: Je größer die Krise im „Ostblock“ war, desto stärker wurde die DDR in den sowjetischen Gravitationsbereich eingebunden. Dies war nach 1948 der Fall, als sich Jugoslawien von der Hegemonialmacht emanzipierte, und auch nach 1956, als Polen und Ungarn diese Richtung einschlugen, oder 1961, als der Abzug der sowjetischen Berater aus der Volksrepublik China im Vorjahr und die darauf folgende fristlose Kündigung von mittelfristig fest eingeplanten Handelsverträgen den „Ostblock“ in eine schwere Wirtschaftskrise stürzten. Auf den ersten Blick schien paradoxerweise Isolation und Schwäche der DDR ihren integrativen Funktionswert zu erhöhen, doch in Wirklichkeit war es unter Chruschtschow umgekehrt die Strukturschwäche des Ostblocks, die seitens der DDR politische, wirtschaftliche und ideologische Integrationsimpulse auslöste und entsprechende Initiativen begünstigte. Da für die DDR die Integration in den Ostblock von existenzieller Bedeutung war, wurden solche Bemühungen von Moskau gefördert.

71 Vgl. Filitow, Alexei: Die Entscheidung zum Mauerbau, Manuskript, Bl. 6.

72 Ebenda, Bl. 7. 
